This is the Accepted Manuscript of an article whose final and definitive form has been published in Neurocase DOI:10.1080/13554794.2014.974620

http://www.tandfonline.com/doi/abs/10.1080/13554794.2014.974620

\title{
Peculiar body representation alterations in hemineglect: a case report
}

Authors: Antonella Di Vita ${ }^{a}$, Liana Palermo ${ }^{\text {a,b }}$, Laura Piccardi ${ }^{\text {ac }}$, Cecilia Guariglia a d

Affiliations:

${ }^{a}$ I.R.C.C.S. Fondazione Santa Lucia, Rome, Italy

${ }^{\mathrm{b}}$ School of Life \& Health Sciences, Aston University, Birmingham UK

${ }^{\mathrm{c}}$ Dipartimento di Medicina Clinica, Sanità Pubblica, Scienza della Vita e dell'Ambiente, Università degli Studi di L'Aquila, L'Aquila, Italy

d Dipartimento Psicologia 39, Sapienza Università di Roma, Rome, Italy

Running Head: Body schema deficit and delusional ideas: a case

\section{Corresponding author:}

Dr. Antonella Di Vita, Psy.D.

Neuropsychology Unit

I.R.C.C.S. Fondazione Santa Lucia

Via Ardeatina, 306

00179 Rome, Italy

mail: antonella.divita@uniroma1.it

Tel. $+39(0) 651501117$

Fax $+39(0) 651501366$ 


\begin{abstract}
We report the case of FP affected by personal and extrapersonal neglect and a body representation deficit characterized by delusional ideas. When FP performed the human figure, he placed body parts to the left, despite his extrapersonal neglect. Differently, when he performed the car figure he placed all parts to the right, in line with his deficit. Comparing FP with a small patient group with the same clinical features without delusional ideas about body emerged that he was the only one to suffer from a specific body representation deficit characterized by a lack of body ownership sense.
\end{abstract}

Keywords: personal neglect, body representation, body ownership, body schema, anosognosia 


\section{Introduction}

Understanding the mechanisms through which the brain represents the body is one of the most challenging issues of neuroscience in the recent years. Our body is, in fact, a special subject of investigation since the ways in which we deal with it are numerous: touch, vision, proprioception, motor behaviour, semantic comprehension, emotions and feelings. So, we receive more information on our body than on any other objects, and unlike other physical objects, we perceive it from within (de Vignemount, 2011).

The different experiences through that we deal with our body explain the wide ranges of body representation alterations following brain injury (for a classification see de Vignemount et al., 2010). Among these disorders, clinical neuropsychology distinguishes between body parts localization (personal neglect; autotopagnosia, etc...) and body awareness (somatoparaphrenia; supernumerary limbs syndrome). Concerning, in particular, personal neglect there is not agreement about its nature as body mental representation deficit. Coslett (1998) showed, for example, that unilateral neglect can be associated with a body schema deficit. Using a task similar to that used by Parson (1994), which required judging whether the presented image of a hand was a left or a right hand, Coslett found that only neglect patients failed to correctly identify the drawings of the left hand.

Guariglia and Antonucci (1992) described a patient who suffered from pure personal neglect who could not correctly locate different ipsilesional and contralesional body parts in reconstructing the image of the body or the face.

Moreover, Baas et al. (2011) compared the performance of patients with and without personal neglect in mental rotation tasks of hands and objects to assess the cognitive mechanisms underlying personal neglect. Their results showed that patients with personal neglect make more errors with left stimuli regardless of whether the stimulus is a hand or an 
object. Furthermore, they found that errors in judging left hands predict the presence of personal neglect, whereas other variables, such as the presence of sensory or motor deficits, were not predictive. Although results of the mental rotation tasks showed that personal neglect could not be attributed only to a deficit in the representation of the body, according to the authors, results of the regression analysis, suggested that the deficit in the representation of the contralesional body was the main mechanism underlying personal neglect.

As mentioned above we deal with our body in different way but what is unique and differs our body from other objects is its ownership, in other words, "the feeling that my body belongs to me, and is ever present in my mental life" (Tsakiris, Hesse, Boy, Haggard, \& Fink, 2007), the body awareness deficits are, in fact, considered a part from body localization deficits in the clinical neuropsychology (see above). The sense of body ownership may be destroyed after a brain damage, this causes different delusional beliefs concerning the body which are usually reported in literature with the term of "somatoparaphrenia". This neurologically deficit is originally defined by Gerstmann (1942) as characterized by 'illusions or distortions concerning the perception of, and confabulations or delusion referring to the affected limbs or side'. Moreover the author distinguished somatoparaphrenia from autosomatamnesia and autosomatagnosia, respectively the absence of memory and consciousness of the affected body parts. Over time many different definitions have been proposed (see Vallar \& Ronchi 2009 for a review) due to the fact that somatoparaphrenia is usually associated with other symptoms (i.e. neglect, anosognosia, somatosensory deficits etc.) and is fluctuating over time (Vallar \& Ronchi 2009). Recently, Vallar \& Ronchi (2009) suggested that defective multisensory integration together with a disorder in the spatial representation of the body is the mechanism at the basis of somatoparaphrenia. Also the neurocognitive model developed by Tsakiris (2010) on the basis of data on rubber hand illusion, suggests that body-ownership originates from the 
interaction between current multisensory input and internal models of the body.

In the present paper we described a patient with a modification at the level of the visuospatial body map that seems related to a lack of body ownership sense and a personal neglect as a consequence of a large lesion in the right hemisphere, involving also the insularlenticular region.

\section{Case History}

FP is a 63-year-old right-handed retired man with 5 years of education who had a vascular accident that resulted in left hemiplegia. He was admitted to the IRCCS Fondazione Santa Lucia (Rome, Italy) for inpatient rehabilitation treatment about ten days after onset of the cerebro-vascular accident (CVA).

The neurological exam at admission showed psychomotor slowing, a tendency towards drowsiness, gaze deflected to the right, dysarthria, facio-brachio-crural left hemiplegia, left limb hypotonia and poor control of the torso in a sitting position.

Visual field assessment performed about one month after the CVA also showed a probable left-sided deficit and a severe visual acuity deficit in the right eye.

Approximately one month after the CVA, an MRI exam was performed at the Neuroimaging Laboratory of the IRCCS Fondazione Santa Lucia. The exam was carried out in a $3.0 \mathrm{~T}$ scanner (Allegra, Siemens Medical Solutions, Erlangen, Germany). The images showed the presence of a large area of altered intensity, hyperintense on $\mathrm{T} 2$ and hypointense on $\mathrm{T} 1$, located in the right cortico-subcortical fronto-temporo-parietal and right insular cortex. Moderate reduction in amplitude of the signal in the subarachnoid spaces adjacent to the mantle and a slight compressive effect on the ipsilateral supratentorial ventricular system, without midline shift, were observed (see Figure 1)

---insert Figure 1 about here---

About twenty days after onset of the CVA, FP underwent an extensive neuropsychological 
evaluation (see Table 1 for details) that revealed the presence of personal and peripersonal neglect.

---insert table 1 about here---

The patient was unable to describe his clinical conditions and was also unaware of his motor deficits (anosognosia for the motor deficit $=2$; Bisiach et al.'s scale: 1986); he spontaneously complained of a generalized state of fear and reported difficulty in speech (dysarthria) and in maintaining attention only if specifically questioned.

FP was also unaware of his explorative deficit in both personal and extrapersonal space.

His wife reported that he had expressed delusional ideas about the left side of his body, i.e., he asked his wife to check whether his arm had been left in the gym because it was no longer there. Following Vallar and Ronchi's (2009) classification, his behaviour corresponded to "delusional beliefs of dis-ownership of the affected body parts". His delusional ideas and anosognosia for neglect and motor deficits fluctuated (this is common in these disease that usually lasts for days or weeks, in fact to our knowledge only three cases of chronic somatoparaphrenia were described, see Cogliano, Crisci, Conson, Grossi \& Trojano, 2012 and Nightingale, 1982). In general, FP felt that his arm was missing; when the examiner pointed out that FP's arm was attached to his shoulder, he stated that it was not his arm. It was not possible to examine in a standard way the characteristic of FP's delusional beliefs since, as mentioned above, these fluctuated over the time.

To more thoroughly analyse this disorder, FP was submitted to an extensive neuropsychological evaluation. The study was approved by the local Ethics Committee in accordance with the Helsinki Declaration and the patient provided his written consent to participate in the neuropsychological investigation.

The patient's visual-explorative abilities were further investigated with a functional evaluation to determine whether he showed any difference between peri-personal and extrapersonal space and with structured tests to assess whether he also had a deficit in the 
imagery domain (representational neglect).

The functional evaluation consisted of a visual-spatial scanning task (TSVs; Judica, Galati, \& Zoccolotti, 1996) in which the patient had to respond as quickly as possible to visual stimuli presented on a wide screen (surface stimulation at $80^{\circ} \times 45^{\circ}$ ); 48 stimuli were presented in random positions. Results showed that FP's exploration of extrapersonal space was strongly asymmetric because he missed most of the contralesional stimuli. The presence of asymmetries in generating an image from long-term memory was assessed by asking the patient to perform the O'clock test (Grossi, Modaferri, Pelosi, \& Trojano, 1989) and the Familiar Square Description test (Guariglia, Palermo, Piccardi, Iaria, \& Incoccia, 2013). The familiar squares chosen for the test were well-known to FP before the illness onset and they were comparable for familiarity and number of details. FP's performance on the O'clock test was well within the normal range ( $L Q=-12$, Cut-off=/> -20 ; Guariglia et al., 2013). Also, on the Familiar Square Description test he made a very detailed description (without any asymmetry) of both left-sided and right-sided elements (Guariglia et al., 2013). Note, however, that FP was unable to maintain the perspective given by the examiner and he used the previously described single elements as a point of reference in recalling the location of the others (e.g. "at the left of the church there are some arches, at the left of the arches is the police station, [...]" etc...). Based on these results, FP showed no signs of representational neglect, but seemed to be affected by a more general deficit in imagining something with respect to his body (i.e. egocentric perspective).

In summary, FP showed severe left unilateral neglect in personal, peri-personal and extrapersonal space but not in the imaginative domain. He also suffered from pervasive somatoparaphrenia and anosognosia. Since, FP showed body representation deficits we submitted him to an extensive evaluation about body representation to better understand the nature of his disorders that if not rehabilitated could compromise his motor recovery. 


\section{Body representation assessment}

Several tests were performed to assess the involvement of different types of body representation in personal neglect.

\section{Human figure drawing}

When FP was asked to draw a human figure, he drew a man in uniform with his head turned to the left (see Figure 2). Although the drawing contains all body parts and their proportions are generally respected, there is evidence of an asymmetry in the height of the shoulders and the length of the lower limbs.

----insert Figure 2 about here---

\section{Localisation of body parts}

The ability to locate body parts was assessed by means of a modified version of Semenza and Goodglass's Test (Semenza \& Goodglass, 1985). In this test, patients are required to point to 26 parts of the body, first on themselves and then on the examiner; body parts are presented randomly and are non-lateralized (i.e., the nose), left-sided and right-sided (i.e., the left eye, the right eye). FP performed flawlessly on both his own and the examiner's body.

\section{Body representation test}

The visuo-spatial map of the body was assessed with the "Frontal body-evocation" (FBE) "Frontal face-evocation" (FFE) and "Lateral face-evocation" (LFE) subtests of the Body Representation test (Daurat-Hmeljak, Stambak, \& Berges, 1978). Although this test was developed for use with children, it has been used to study impairments of body representation in brain-damaged patients (Canzano, Piccardi, Bureca, \& Guariglia, 2011; Guariglia \& Antonucci, 1992; Guariglia, Piccardi, Puglisi Allegra, \& Traballesi, 2002; Marangolo, Piccardi, Rinaldi, 2003). The test materials include a small plastic board on which one reference part (the head for FBE, the contour of the face for FFE, and hair for 
LFE) is depicted, and several tiles that represent body parts. Participants are presented with one tile at time and their task is to name the body part depicted on the tile before putting it on the board. Before presenting a new tile, the position of the previous tile is recorded on an overlapping grid and the tile is removed. Moreover, for the LFE subtest the subject has to choose among different views of the same body part before putting the tile on the table; for example, the nose is presented in frontal, lateral right and lateral left views. Given that the original test proposes only the right profile for the LFE subtest, an analogous version of the left profile was created to determine whether the patient had any asymmetries due to the presence of personal neglect.

FP's results on each sub-test for both recognition (naming) and localization of the different body parts are presented in Table 2 .

----insert table 2 about here---

A descriptive analysis of the patient's performance showed that when he performed the subtest which evaluated representation of the whole body (FBE) FP put all the body parts on the left side of the board despite his severe left neglect (see Figure 3). This amazing behaviour was observed only on the FBE subtest, not on the FFE or in the LFE (see Figure 4).

---insert Figure 3 about here---

---insert Figure 4 about here---

The presence of a deficit in body representation was confirmed not only by the localization errors FP made in all the sub-tests, but also by his choice errors on the LFE subtest. For example, FP often chose frontal view parts instead of lateral ones, right parts instead of left ones, and left parts instead of right ones.

Since performance of the Body Representation test (Daurat-Hmeljak et al., 1978) can be affected by the presence of visual-constructive deficits, constructional apraxia was assessed 
using copy of the Rey Complex Figure (Carlesimo et al., 2002) and the Constructional Apraxia test (Spinnler \& Tognoni, 1987).

On both tests, FP's performance was clearly affected by the presence of neglect; that is, he omitted the left-sided parts of the models and under-utilized the left side of the sheet of paper. The presence of neglect emerged also in the strategies used to reproduce the models that strongly disadvantaged his performance. Taking into account his exploratory deficit, he performed in the normal range (raw score: $8 / 14,14$ standard score=1) on the Constructional Apraxia test (Spinnler \& Tognoni, 1987) and defectively on the copy of Rey's Complex Figure (raw score: 9/36, standard score=0; Carlesimo et al., 2002).

To evaluate the presence of general deficits in selecting and localizing parts of noncorporeal objects, we also administered the Car test (Guariglia et al., 2002), which was developed to compare performance on the Body Representation test (Daurat-Hmeljak et al., 1978) with performance on a non-body object that had well-defined right and left sides and involved procedures and materials similar to those of Daurat-Hmeljak et al.'s test. In this test, FP detected and correctly named 5/12 parts of the car and correctly placed 4/12 parts. Errors consisted mostly of mis-locations of the parts. In fact, consistently with his neglect for peri-personal and extrapersonal space, FP placed the parts mostly in the right space (see Figure 5).

---insert Figure 5 about here---

A comparison of the patient's performance on the Body Representation Test and the Car Test showed that FP tended to use only half of the plastic board to locate single parts but, very surprisingly, used opposite sides for the two tests; that is, coherently with the presence of left hemineglect in peri-personal and extra-personal space, FP used only the most rightsided part of the board to locate parts of the non-corporeal objects in the Car test, but used only the most left-sided part of the board when he placed body parts in the FBE sub-test (see Figure 3). Such incoherent use of just half of the board depending on the nature of the 
stimulus (body/non-body) has never been reported before. Therefore, we compared FP's performance on the FBE subtest with the performance of 13 healthy control participants (C) and 5 right brain-damaged patients (BDP) suffering from both personal and peripersonal neglect (see Table 3 for neglect assessment details) but not by somatoparaphrenia and/or representational neglect.

---insert Table 3 about here---

In particular, using BTD_Cov.exe software (Crawford, Garthwaite, \& Ryan, 2011), the number of parts properly recognized (recognition score-R), parts properly positioned (localization score-L) and left body parts placed to the right (left-right inversion error scoreLRI error) by FP were compared to those of $\mathrm{C}$ and BDP. Since only $\mathrm{C}$ (not BDP) were matched for age and education, these factors were used as covariates (Table 4 shows the means and standard deviations of the two groups and FP's performance).

---insert table 4 about here---

Results show that FP's recognition performance differed significantly from the C group's ( $p$ $=0.03)$ but not the BDP group's $(\mathrm{p}=0.46)$ performance.

The same pattern of results emerged when the number of parts correctly positioned was compared: also in this case, FP scored lower than the $\mathrm{C}$ group $(\mathrm{p}=0.03)$ but not the BDP group $(p=0.44)$. Instead, FP's performance differed significantly from that of both $\mathrm{C}(\mathrm{p}=$ $0.00)$ and $\mathrm{BDP}(\mathrm{p}=0.026)$ groups for number of left-right inversions (LRI).

In summary, the patient's performance was significantly different from the C group's performance on all indices considered, but differed significantly from the BDP group's performance only in the number of LRI errors, because in the latter group LRI errors were infrequent.

Note that a perusal of individual patients' performances shows that no patients in the BDP group performed the test by locating parts only on the left half of the board. 


\section{Lesion analysis}

\section{Subtraction Analysis}

In order to identify the anatomical structures damaged in FP but not in the BDP group an exploratory Subtraction Analysis was conducted.

The boundaries of the lesions were delineated directly on the Modified Driven Equilibrium Fourier Transform (MDEFT) for every single axial slice using MRIcron (Rorden, Karnath, \& Bonilha, 2007; www.Mricro.com/mricron) software. For each lesion, the corresponding mask was created. The volume of the lesion and the full set of MDEFT sequences were simultaneously normalised according to the protocol of Brett, Leff, Rorden, \& Ashburner (2001) by using SPM8 (Welcome Department of Cognitive Neurology, London, UK) software implemented in Matlab (The MathWorks Inc., Natick, MA, USA). Then, using MRIcron software (Rorden et al., 2007) a lesion overlap image of three patients in the BDP (two patients of the BDP group did not agree to undergo the MRI exam) group was created. FP's lesion was overlapped with that of a patient with personal neglect (Pt6) who commits only an LRI error. It should be noted that Pt6 was not considered in the previous analyses because the patient was affected only by personal neglect.

The results presented in Figure 6 indicate the areas that are damaged in patients who made LRI errors in the FBE sub-test; these include the right insula, precentral gyrus and superior, middle and inferior frontal gyrus. The lesions also include the white matter surrounding the above-mentioned structures and the superior corona radiata.

$$
\text { ---insert Figure } 6 \text { about here--- }
$$

\section{Discussion}

The case of FP provides new insights about the complex body representation process.

Different hypotheses can be advanced to explain the patient's peculiar performance on the neuropsychological tests. First, it can be hypothesized that to carry out the task of locating 
body parts on Daurat-Hmeljak et al's test FP had to access the mental representation of his own body which, due to the presence of personal neglect, is distorted. Due to this distorted representation, FP is unable to correctly locate left-sided body parts but is still able to locate right-sided ones. Thus, when required to locate the right arm on the board, FP correctly put it on the left side; but when required to locate the left arm, his distorted body representation did not allow FP to put it on the right half of the board, corresponding to the left side of the body.

The left side of space is, in fact, the right side of the body because the figure is presented in a frontal view. This "mirroring" error in locating body parts, but not non-body parts, cannot be attributed to the mere presence of personal neglect. As found also in previous studies in which the same FBE task was used (Guariglia \& Antonucci, 1992; Piccardi, Nico \& Guariglia poster session, 2002), BDP patients showed no asymmetry on the FBE task but made errors in localizing body parts on both sides of space. These data support the hypothesis that personal neglect is a disorder of the mental representation of the body (Baas et al., 2011; Palermo, Di Vita, Traballesi, \& Guariglia, 2014). Nevertheless, FP's performance was peculiar and rather different from that of previous patients affected by the same body representation deficit who were never reported to neglect the right side of the board in the FBE task. The absence of other forms of neglect could account for the performance differences between EDS (Guariglia \& Antonucci, 1992) and FP. Indeed, it must be considered that the patient described by Guariglia and Antonucci (1992) and the patients reported by Piccardi Nico \& Guariglia (poster session, 2002) suffered from pure personal neglect, whereas FP was affected by both personal and extrapersonal neglect. However, as all of the BDP patients recruited for the present study were affected by both personal and extrapersonal neglect, the possibility that they used one half of the board for body representation (FBE test) and the other half for object representation (Car Test) has to be excluded, because this peculiar behaviour was not observed in any patient except FP. 
Furthermore, analysis of the errors excludes the possibility of attributing FP's inversion errors exclusively to the co-presence of extrapersonal neglect. Indeed, localization errors were observed in both BDP and FP, with the same frequency as that reported in previous studies; it should be noted that FP's localization performance did not differ from that of BDP (Guariglia \& Antonucci, 1992; Piccardi Nico \& Guariglia (poster session, 2002). The only difference was the high number of inversion errors made by FP, because this type of error is infrequent in BDP and never described in previous studies (Guariglia \& Antonucci, 1992; Piccardi Piccardi Nico \& Guariglia (poster session, 2002).

Apart from FP's many inversion errors, what makes him different from the patient described by Guariglia and Antonucci (1992), the patients reported by Piccardi Nico \& Guariglia (poster session, 2002) and the patients in the BDP group is the presence of a delusional idea about the body.

The lack of ownership of the left side of the body, which characterized FP, could explain his performance on the Body Representation test. When the task required an embodiment ("the sense of being localized within one's physical body" - Arzy, Thut, Mohr, Michel, \& Blanke, 2006), similar to the FBE sub-test, FP showed a half body representation lacking of the left body parts placed on the right of the space.

The presence of an altered visuo-spatial body representation could also explain the patient's difficulty in maintaining his egocentric frame of reference during the description of the familiar squares. Indeed, an embodiment is required to perform this task. In order to build up an egocentric representation of the square and properly describe it, the subject has to imagine his own body with respect to the environment. As FP was unable to represent his own body, he could not use this representation as the reference point to mentally explore the represented square and to describe the elements in the square and had to use the spatial relations among the elements.

In the light of this interpretation, FP's good performance on the modified version of 
Semenza and Goodglass's test deserves further explanation. His performance indicates that he was able to localise body parts on both his body and that of the examiner. Being able to point to body parts might depend on an embodiment process (e.g., I can localize your right eye because of my embodiment in you). Indeed, Auclair, Noulhiane, Raibaut and Amarenco (2009) interpreted their case of heterotopoagnosia as an embodiment deficit. In their patient, the impairment in converting the intrinsic spatial coding of the body parts into extrinsic ones was revealed by difficulty in pointing to body parts both on the examiner and a schematic body representation. Nevertheless, the same task can be performed by relying on other types of body representation, such as semantic representation of the body (e.g., having semantic knowledge about the right eye I am able to recognize it and to point to it even if I have no idea about its actual location). Indeed, FP did not lose his semantic knowledge of the body. He never failed to recognize single body parts. Furthermore, the modified version of Semenza and Goodglass's Test has a strong categorical nature that helped FP use his preserved body knowledge. In fact, pointing to single body parts by relying on semantic processing does not necessarily require knowledge about the mutual position of different body parts; thus, people are able to recognize a right ear or a left knee even if they are presented in isolation or drawn in the wrong place (which happens when we look at a cubist portrait).

In the literature, other cases of correct pointing to body parts that were the object of delusional ideas were reported by Meador, Loring, Feinberg, Lee, and Nichols (2000). In their study, the presence of asomatoagnosia ("i.e., the inability to recognize the affected limb as one's own") was not related to non-verbal knowledge (pointing) to the location of a paralyzed limb; in fact, patients who correctly pointed to the paralyzed limb were affected by asomatoagnosia.

Theoretical frameworks of body representation, like the triadic taxonomy of body representation (Schwoebel \& Coslett, 2005; Sirigu, Grafman, Bressler, \& Sunderland, 1991) 
considers the existence of a triadic representation of the body: i) the "body schema" a sensory-motor representation of the body; ii) the "semantics of the body", a conceptual and linguistic representation, and iii) the "structural description of the body," that is a visuospatial map of the different body parts. According to this FP shows a mental body representation deficit at the level of the visuo-spatial body map. This deficit seems to explain both the presence of personal neglect and the lack of ownership of his left limbs. In this view, the FP's performance on the FBE sub-test confirms the hypothesis that somatoparafrenic delusion has a spatial component (Vallar \& Ronchi, 2009) which is framed at the visual-spatial body map level.

Results of the lesion analysis also confirm the link between spatial body representation and body ownership. Although it should be considered that the analyses were conducted in a small number of patients, the results of the subtraction analysis showed the involvement of brain areas (e.g., the insula and frontal lobe) which are considered part of the network that processes the sense of body ownership. In particular, the insula is considered to constitute the basis for the subjective experience of the body (Tsakiris, 2010) because of its role in elaborating visceral, somatosensory and vestibular information (Augustine, 1996; Craig, 2009). Even if the involvement of the insula in the formation of the sense of limbs ownership emerges from both lesion (Baier \& Karnath, 2008) and PET (Tsakiris et al., 2007) studies, and from the application of the rubber hand illusion paradigm (Moseley et al., 2008; Ehrsson, Wiech, Weiskopf, Dolan, Passingham, 2007), the insula contribution has not yet been definitely determined. Some authors (i.e., Gondola et al., 2012) consider that the insula damage is associated with neglect that is almost invariably present together with somatoparaphrenia.

The insula involvement here reported is in line with those studies that consider the insula important for the body representation due to its contribution in the processing of interoceptive information (Craig, 2009; Grossi, Di Vita, Palermo, Sabatini, Trojano, 
Guariglia, 2014). As Berlucchi \& Aglioti (2010) noted, this information become important not only for the sensations per se but also because these sensations evoke actions directed to the body (i.e., scratch the itchy).

The importance of other information besides the proprioceptive one's in the genesis of the body ownership sense is shown also by the dissociation between sense of disownership and undamaged capacities in judging the position sense described by Cogliano et al. (2012) in a patient with chronic somatoparaphrenia.

Moreover, with regard to the frontal lobe, results show involvement of the premotor cortex, the site for the integration of multisensory information, that interact to build up a consistent representation of the body parts (Gandola et al., 2012). In fact, neuroimaging studies (Ehrsson, Spence, \& Passingham, 2004; Tsakiris et al., 2007) show activation of the premotor cortex during the rubber hand illusion.

In summary, we hypothesise that a crucial role in the visual spatial body map is played by the integration of information conveyed by the insula with other sensory-motor information coming from the body, which is then integrated in the frontal area representing the seat of other bodily representations. Damage in this network results in a lack of this integration and affects the ability to represent the body as a whole. Thus, the body representation becomes that of a half body because the patient is unable to update his body representation with information coming from the right side of the body and the visually perceived contralesional body parts do not elicit any sense of ownership and thus produce the delusional behaviour.

\section{Acknowledgements}

This study was supported by funding from MIUR to C.G. (PRIN 2010-2011; nr 2010ENPRYE_004).

The funders had no role in the study design, data collection and analysis, decision to publish 
or preparation of the manuscript. 


\section{References}

Arzy, S., Thut, G., Mohr, C., Michel, C.M., \& Blanke, O. (2006). Neural basis of embodiment: distinct contributions of temporoparietal junction and extrastriate body area. The Journal of Neuroscience, 26, 8074-8081.

Auclair, L., Noulhiane, M., Raibaut, P., \& Amarenco, G. (2009). Where are your body parts? A pure case of heterotopagnosia following left parietal stroke. Neurocase, 15, 459-465. doi: 10.1080/13554790902911642

Augustine, J. R. (1996). Circuitry and functional aspects of the insular lobe in primates including humans. Brain Research: Brain Research Reviews, 22, 229-244.

Baas, U., de Haan, B., Grässli, T., Karnath, H.O., Mueri, R., Perrig, W.J., Wurtz, P., \& Gutbrod, K. (2011). Personal neglect-a disorder of body representation? Neuropsychologia, 49, 898-905. doi: 10.1016/j.neuropsychologia.2011.01.043.

Baier, B. \& Karnath, H.O. (2008) Tight link between our sense of limb ownership and selfawareness of actions. Stroke, 39:486-488.

Berlucchi G., Aglioti S.M. (2010) The body in the brain revisited. Experimental Brain Research, 200, 25-35.

Bisiach, E., Vallar, G., Perani, D., Papagno, C., \& Berti, A. (1986). Unawareness of disease following lesions of the right hemisphere: Anosognosia for hemiplegia and anosognosia for hemianopia. Neuropsychologia, 24, 471-482. 
Brett, M., Leff, A.P., Rorden, C., \& Ashburner, J. (2001). Spatial normalization of brain images with focal lesions using cost function masking. Neuroimage, 14, 486-500.

Canzano, L., Piccardi, L., Bureca, I., \& Guariglia, C. (2011). Mirror writing resulting from an egocentric representation disorder: a case report. Neurocase, 17, 447-460. doi: $10.1080 / 13554794.2010 .532143$

Carlesimo, G.A., Buccione, I., Fadda, L., Graceffa, A., Mauri, M., Lorusso, S., Bevilacqua, G., \& Caltagirone, C. (2002) Standardizzazione di due test di memoria per uso clinico: Breve Racconto e Figura di Rey. Nuova Rivista di Neurologia, 12, 1-3.

Coslett, H.B. (1998). Evidence for a disturbance of the body schema in neglect. Brain and Cognition. 37, 527-544.

Cogliano, R., Crisci, C., Conson, M., Grossi, D., Trojano, L. (2012) Chronic somatoparaphrenia: a follow-up study on two clinical cases. Cortex, 48, 758-67.

Craig, A. D. (2009). How do you feel-now? The anterior insula and human awareness. Nature Reviews Neuroscience, 10, 59-70. doi: 10.1038/nrn2555

Crawford, J. R., Garthwaite, P. H., \& Ryan, K. (2011). Comparing a single case to a control sample: Testing for neuropsychological deficits and dissociations in the presence of covariates. Cortex, 47, 1166-1178. doi: 10.1016/j.cortex.2011.02.017

de Vignemont F. (2010) Body schema and body image--pros and cons. Neuropsychologia, 48, 669- 
680.

de Vignemont F. (2011) Embodiment, ownership and disownership. Consciouness and Cognition, 20, 82-93.

Daurat-Hmeljak C., Stambak M., \& Berges J. (1978). Il test dello schema corporeo. Una prova di conoscenza e costruzione dell'immagine del corpo. Firenze:Organizzazioni Speciali.

Ehrsson, H.H., Wiech, K., Weiskopf, N., Dolan, R.J., Passingham, R.E. (2007) Threatening a rubber hand that you feel is yours elicits a cortical anxiety response. Proc Natl Acad Sci USA, 104, 9828-9833.

Ehrsson, H.H., Spence, C., \& Passingham, R.E. (2004) That's my hand! Activity in premotor cortex reflects feeling of ownership of a limb. Science, 305, 875-877.

Gandola, M., Invernizzi, P., Sedda, A., Ferrè, E.R., Sterzi, R., Sberna, M., Paulesu, E., \& Bottini G. (2012). An anatomical account of somatoparaphrenia. Cortex, 48, 1165-1178. doi: 10.1016/j.cortex.2011.06.012

Gerstmann, J. (1942) Problem of imperception of disease and of impaired body territories with organic lesions. Relation to body schema and its disorders. Archives of Neurology \& Psychiatry, 48, 890-913.

Grossi, D., Modaferri, A., Pelosi, L., \& Trojano, L. (1989). On the different roles of the cerebral hemispheres in mental imagery: the "O' Clock Test" in two clinical cases. Brain and Cognition, 10, 18-27. 
Grossi, D., Di Vita, A., Palermo, L., Sabatini, U., Trojano, L. \& Guariglia, C. (2014) The brain network for self-feeling: A symptom-lesion Mapping study. Neuropsychologia. doi: 10.1016/j.neuropsychologia.2014.08.004.

Guariglia, C., \& Antonucci, G. (1992). Personal and extrapersonal space: a case of neglect dissociation. Neuropsychologia, 30, 1001-1009.

Guariglia, C., Piccardi, L., Puglisi Allegra, M.C., \& Traballesi, M. (2002) Is autotopoagnosia real? EC says yes. A case study. Neuropsychologia, 40, 1744-1749.

Guariglia, C., Palermo, L., Piccardi, L., Iaria, G., \& Incoccia, C. (2013) Neglecting the left side of a city square but not the left side of its clock: prevalence and characteristics of representational neglect. Plos One, 8, 1-9. doi: 10.1371/journal.pone.0067390

Judica, A., Galati, G., \& Zoccolotti P. (1996). Metodiche per la diagnosi ed il trattamento riabilitativo del paziente eminattento. Centro Ricerche di Neuropsicologia IRCCS S. Lucia. Roma:Edizioni Erre.

Marangolo, P, Piccardi. L., \& Rinaldi, M. C. (2003). Dissociation between personal and extrapersonal neglect in a crossed aphasia study. Neurocase, 9, 414-20.

Meador, K.J., Loring, D.W., Feinberg, T.E., Lee, G.P., \& Nichols, M.E. (2000). Anosognosia and asomatognosia during intracarotid amobarbital inactivation. Neurology, 55,816-820.

Moseley, G.L., Olthof, N., Venema, A., Don, S., Wijers, M., Gallace, A., Spence C. (2008) 
Psychologically induced cooling of a specific body part caused by the illusory ownership of an artificial counterpart. Proc Natl Acad Sci USA, 105, 13169-13173.

Nightingale S. (1982) Somatoparaphrenia: A case report. Cortex, 18, 463-467.

Novelli, G., Papagno, C., Capitani, E., Laiacona, M., Vallar, G., Cappa, S.F. (1986). Tre test clinici di ricerca e produzione lessicale. Taratura si soggetti normali. Archivio di Psicologia, Neurologia e Psichiatria, 47: 477-506.

Orsini, A., (2003) La memoria diretta e la memoria inversa di cifre in soggetti dai 16 ai 64 anni. Bollettino di Psicologia Applicata, 239, 73-77.

Orsini, A., Grossi, D., Capitani, E., Laiacona, M., Papagno, C., \& Vallar G. (1987) Verbal and spatial immediate memory span: normative data from 1355 adults and 1112 children. Italian Journal of Neurological Sciences, 8, 539-548.

Palermo, L., Di Vita, A., Piccardi, L., Traballesi, M., \& Guariglia, C. (2014). Bottom-up and top-down processes in body representation: A study of brain-damaged and amputee patients. Neuropsychology, 28, 772-781.

Parsons, L. M. (1994) Temporal and kinematic properties of motor behavior reflected in mentally simulated action. Journal of Experimental Psychology: Human Perception \& Performance, 20, 709-30.

Piccardi L, Nico D ,\& Guariglia C. (2002, October). Personal neglect and body representation. Poster session presented at the meeting of The Human Brain. 
International conference on the structural basis of understanding human brain function and dysfunction. Rome, Italy.

Pizzamiglio, L, Judica, A, Razzano, C, \& Zoccolotti, P. (1989) Toward a comprehensive diagnosis of visual-spatial disorders in unilateral brain-damaged patients. Psychological Assessement, 5, 199-218.

Rorden, C., Karnath, H.-O. \& Bonilha, L. (2007). Improving lesion-symptom mapping. Journal of Cognitive Neuroscience, 19, 1081-1088.

Semenza, C., \& Goodglass, H. (1985). Localization of body parts in brain injured patients. Neuropsychologia, 23, 161-75.

Schwoebel, J., \& Coslett, H.B. (2005). Evidence for multiple, distinct representations of the human body. Journal of Cognitive Neuroscience, 17, 543-553.

Sirigu, A., Grafman, J., Bressler, K., \& Sunderland, T. (1991). Multiple representations contribute to body knowledge processing. Evidence from a case of autotopagnosia. Brain, 114,629-642.

Spinnler, H., \& Tognoni, G. (1987). Standardizzazione e taratura italiano di test psicologici. Italian Journal of Neurological Sciences; 6 (suppl.8), 1-120.

Tsakiris, M., Hesse, M.D., Boy, C., Haggard, P., \& Fink, G.R. (2007) Neural signatures of body ownership: A sensory network for bodily self-consciousness. Cerebral Cortex, 17, $2235-2244$ 
Tsakiris, M. (2010). My body in the brain: A neurocognitive model of body-ownership. Neuropsychologia, 48, 703-712. doi: 10.1016/j.neuropsychologia.2009.09.034

Vallar, G., \& Ronchi, R. (2009). Somatoparaphrenia: A body delusion. A review of the neuropsychological literature. Experimental Brain Research, 192, 533-551. doi: 10.1007/s00221-008-1562-y

Zoccolotti, P., Antonucci, G., \& Judica, A. (1991). Psychometric characteristics of two semistructured scales for the functional evaluation of hemi-inattention in extrapersonal and personal space. Neuropsychological Rehabilitation, 2, 179-191. 


\section{Figure legends:}

Figure 1. Sagittal (a) and axial (b) section of FP's MR
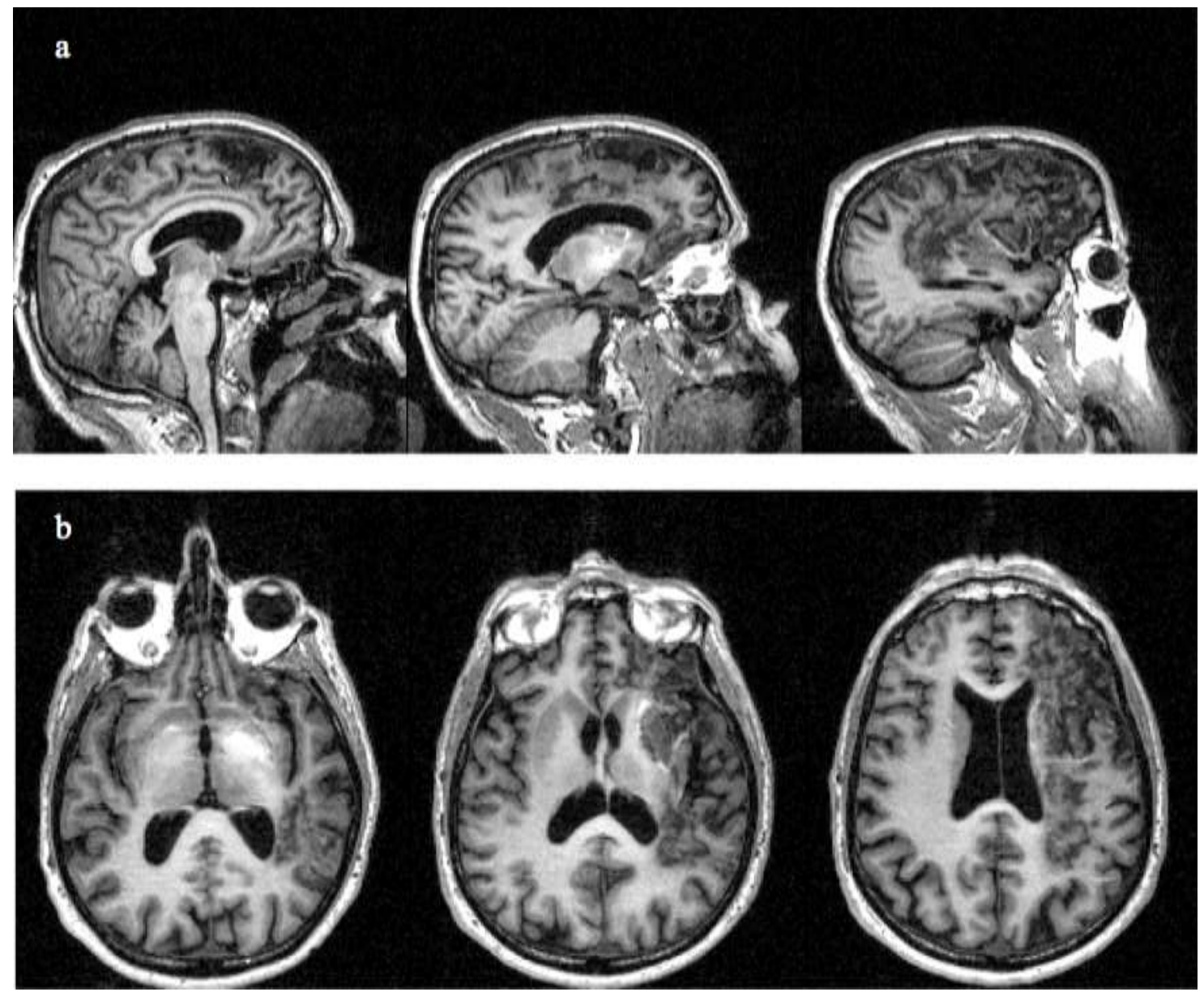
Figure 2. Human Figure drawn by FP

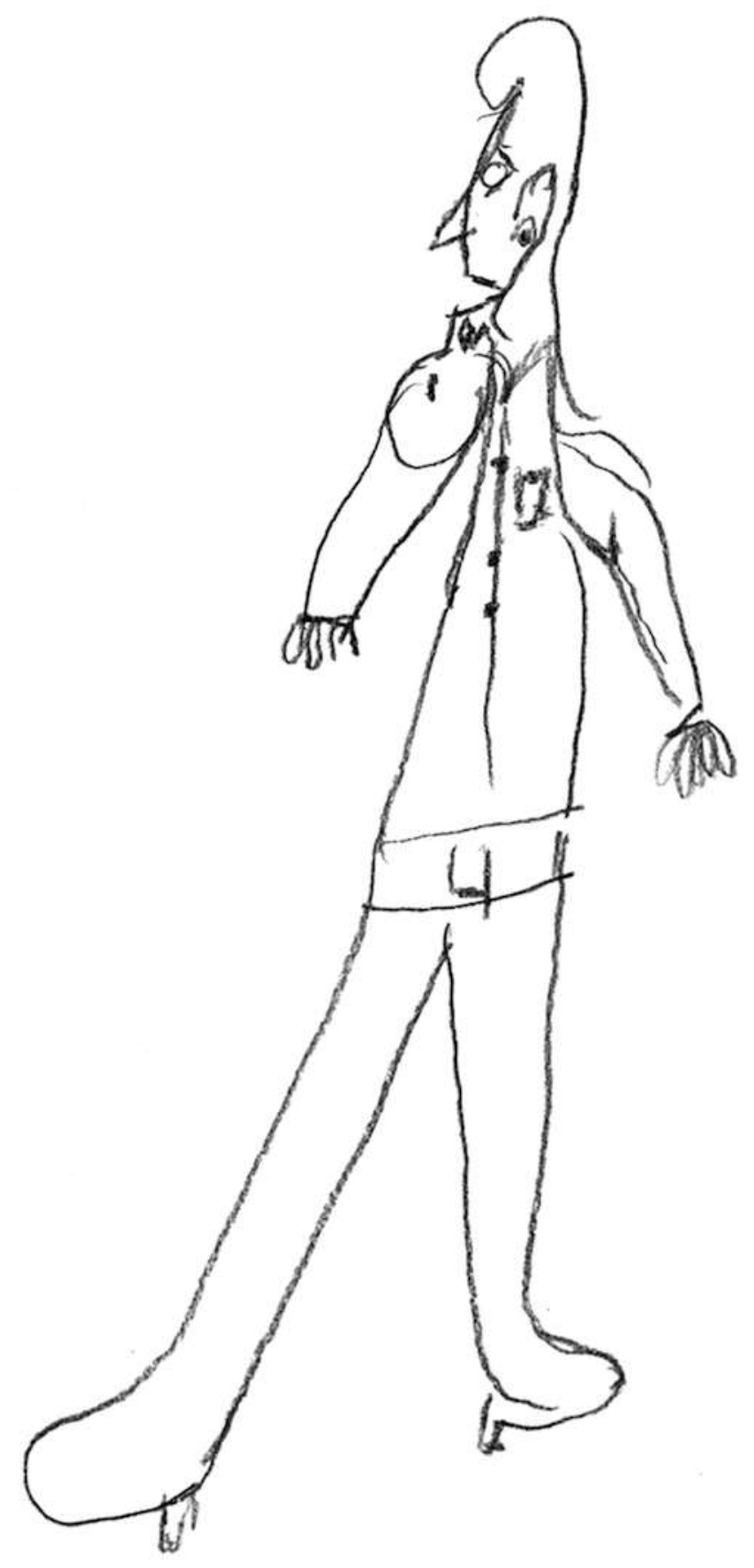


Figure 3. Performance on the Frontal body-evocation sub-test of FP (a) and that of a patient of the BDP group (b)

a

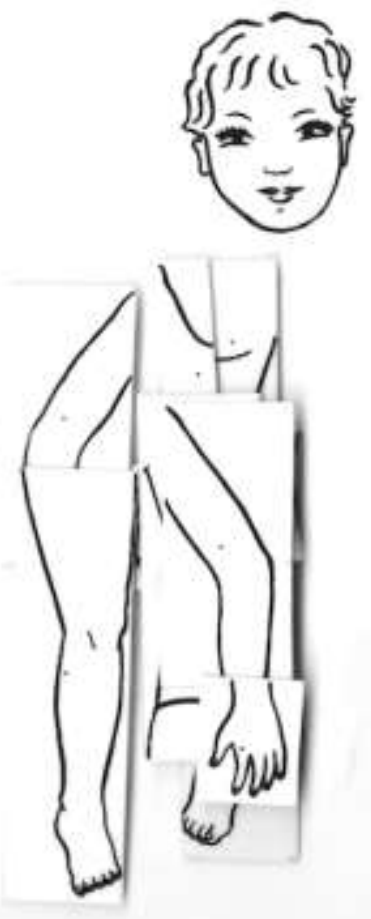

b

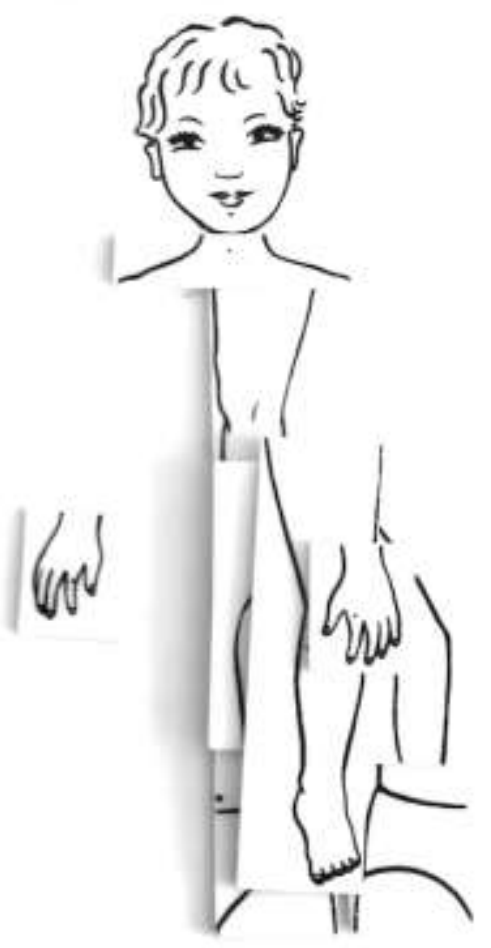


Figure 4. FP's performance on the Frontal face-evocation sub-test (a), Lateral face-evocation (right) sub-test (b) and Lateral face-evocation (left) sub-test (c) of the Body Representation Test.
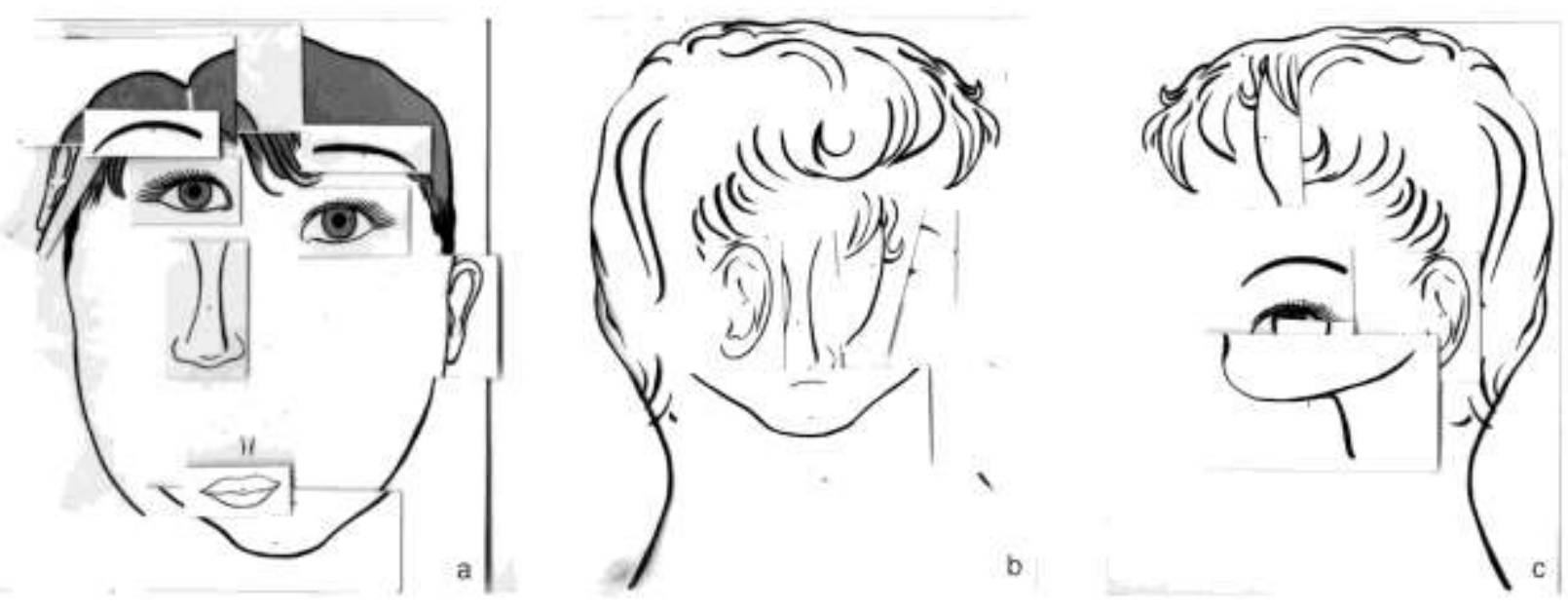
Figure 5. Car Test

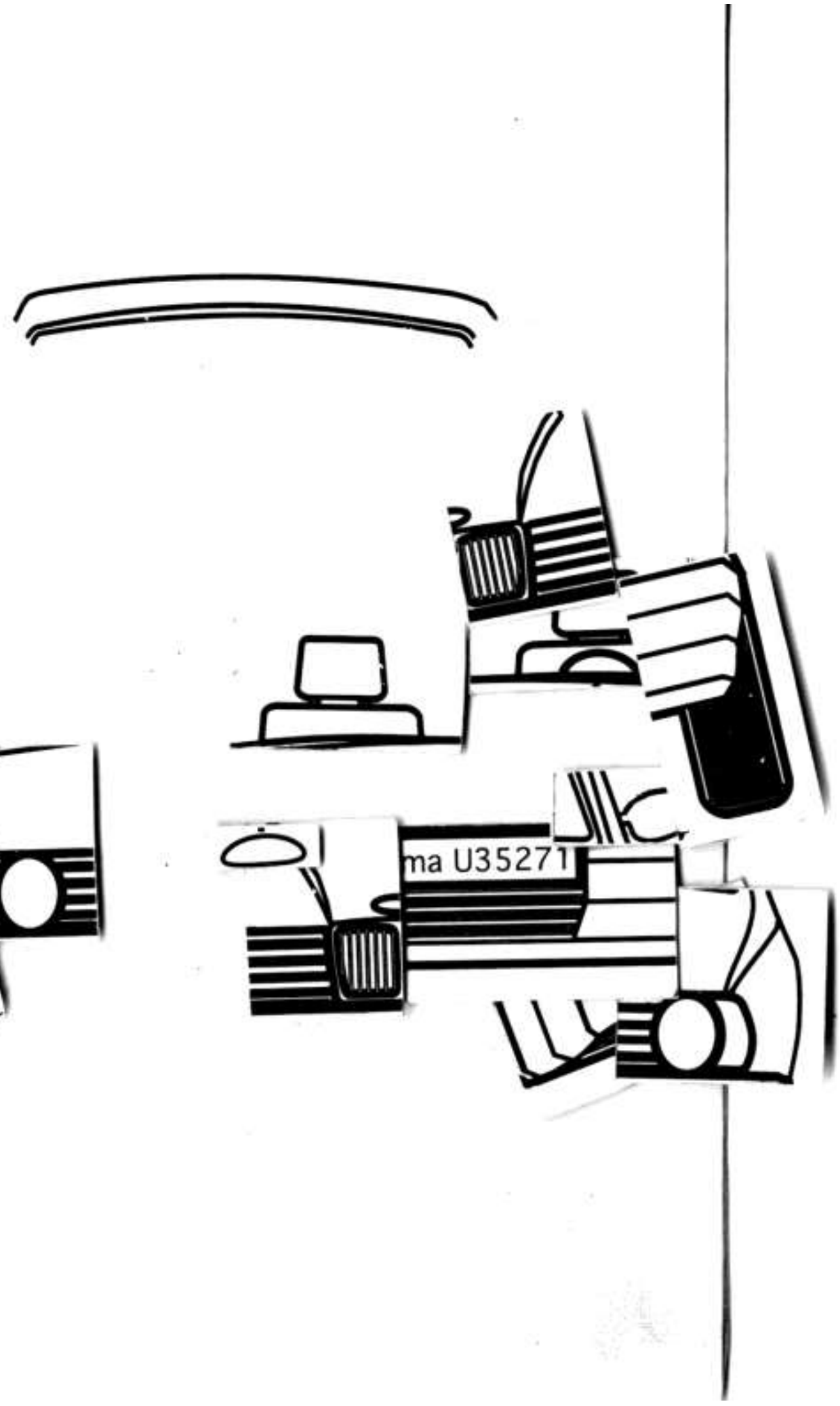


Figure 6. Subtraction Analysis. The figure shows the probability map resulting from subtraction of the probability map of the patients who made LRI-errors from the probability map of patients who did not make LRI-errors. The range of colours from 30 indicates areas with the greatest probability of being damaged in patients who make LRI-errors.

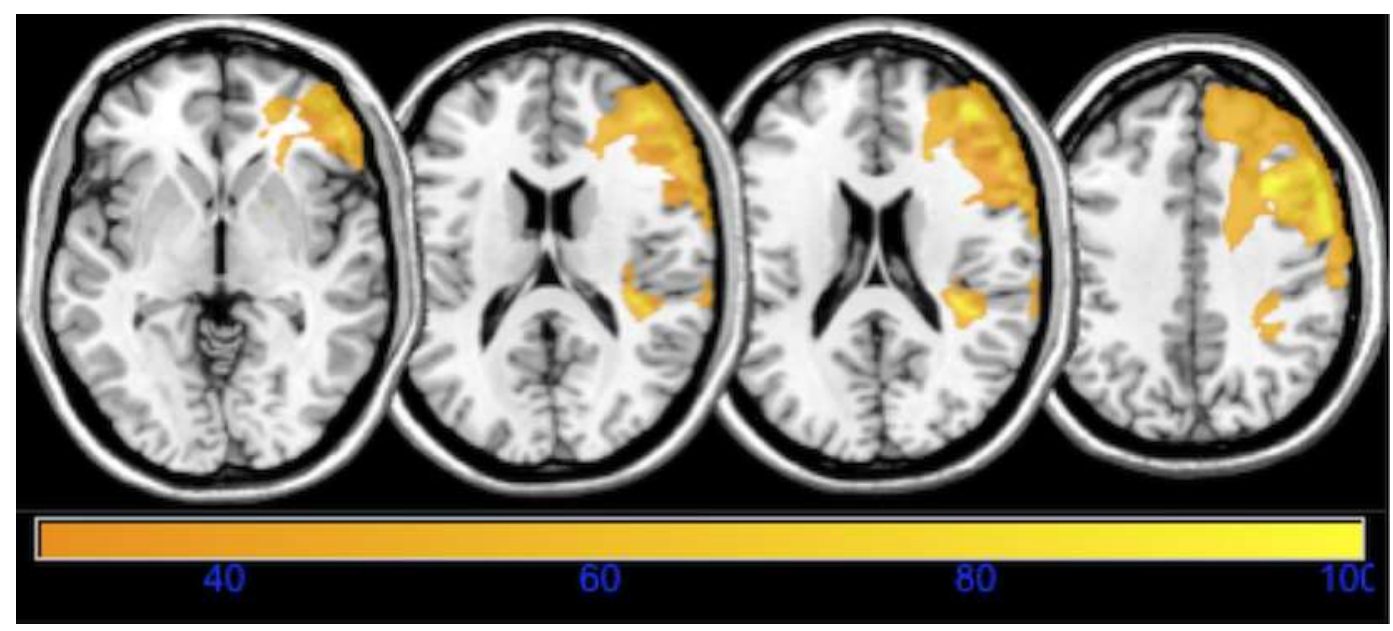




\begin{tabular}{|c|c|c|}
\hline TEST & FP score & SS* \\
\hline Time Orientation (Spinnler \& Tognoni, 1987) & $96 / 100$ & \\
\hline Space Orientation (city/hospital/hospital name) & +++ & \\
\hline Verbal Judgment (Spinnler \& Tognoni, 1987) & $46 / 60$ & 3 \\
\hline Episodic memory (Novelli et al., 1986) & $13,5 / 28$ & 4 \\
\hline Digit Span ( Orsini et al.,1987) & $6 / 9$ & 4 \\
\hline WAIS Digit Span Backward (Orsini, 2003) & 3 & 7 \\
\hline \multicolumn{3}{|l|}{$\begin{array}{l}\text { Standard Battery for the Evaluation of Hemineglect** (Pizzamiglio et al., } \\
\text { 1989) }\end{array}$} \\
\hline Letter Cancellation test & $\begin{array}{l}\text { SN 0/53 } \\
\text { DX 2/51 }\end{array}$ & \\
\hline Line Cancellation test & $\begin{array}{l}\text { SN 0/11 } \\
\text { DX 1/10 }\end{array}$ & \\
\hline Wundt-Jastrow Area Illusion test & $\begin{array}{l}\text { SN 8/20 } \\
\text { DX 0/20 }\end{array}$ & \\
\hline Sentence Reading test & $0 / 6$ & \\
\hline Personal Neglect $* * *$ (Zoccolotti et al.,1991) & 9/9 & \\
\hline
\end{tabular}

Performances below the cut-off are indicated in bold

*SS = standard score from 1 to 4 in the normal range; 0 is pathological

**Patients who scored below the cut-off on at least two of these four tests were considered to have visuo-spatial neglect (Pizzamiglio et al., 1989). Letter Cancellation test: cut-off is a difference of 4 (or more) omissions between the right and the left side; Line Cancellation: cut-off is $>2$ omissions on the left side; Wundt-Jastrow Area Illusion Test: cut-off is a difference $>2$ between unexpected answers (i.e., responses in the direction opposite to the illusory effect observed in healthy individuals) given for left-oriented minus right-oriented stimuli; Sentence Reading Test: Hits are the number of correctly read sentences. The cut-off is one or more sentences incompletely read on the left side.

*** The presence of personal neglect was revealed by means of a standardized test (Zoccolotti et al., 1991) that required the use of three objects(eyeglasses, a razor or powder and a comb) in the body space. For each object, the examiner assigned a score based on the asymmetry in performance of the patient in the left and right space $(0=$ no asymmetry, $3=$ maximal asymmetry $)$. Cut-off $\geq 2$ 
Table 2 FP's performance on the Body Representation sub-tests

\begin{tabular}{r|c}
\multicolumn{1}{c|}{ SUB-TEST } & FP score \\
\hline \multicolumn{1}{c|}{ Frontal body-evocation } & \\
\hline Recognition & $6 / 9$ \\
\hline Frontal face-evocation & $2 / 9$ \\
\hline Recognition & $11 / 12$ \\
\hline Localization & $2 / 12$ \\
\hline Lateral face-evocation (right) & \\
\hline Face part choice & $1 / 6$ \\
\hline Recognition & $6 / 6$ \\
\hline Localization & $0 / 6$ \\
\hline Lateral face-evocation (left) & \\
\hline Face part choice & $5 / 6$ \\
\hline Recognition & $4 / 6$ \\
\hline Localization & $1 / 6$ \\
\hline
\end{tabular}


Table 3 Neglect Assessment in the BDP group and Pt6

\begin{tabular}{|c|c|c|c|c|c|c|c|c|}
\hline \multirow[t]{3}{*}{ Participants } & \multicolumn{7}{|c|}{ Standard Battery for the Evaluation of Hemineglect** } & \multirow{2}{*}{$\begin{array}{c}\text { Personal } \\
\text { Neglect*** } \\
(\max 9)\end{array}$} \\
\hline & \multicolumn{2}{|c|}{ Letter Cancellation } & \multicolumn{2}{|c|}{ Line Cancellation } & \multicolumn{2}{|c|}{$\begin{array}{c}\text { Wund-Jastrow illusion } \\
\text { test }\end{array}$} & $\begin{array}{c}\text { Sentence } \\
\text { Reading test } \\
(\max 6)\end{array}$ & \\
\hline & $\begin{array}{c}\text { Left } \\
(\max 53)\end{array}$ & $\begin{array}{c}\text { Right } \\
(\max 51)\end{array}$ & $\begin{array}{c}\text { Left } \\
(\max 11)\end{array}$ & $\begin{array}{l}\text { Right } \\
(\max 10)\end{array}$ & $\begin{array}{c}\text { Left } \\
(\max 20)\end{array}$ & $\begin{array}{l}\text { Right } \\
(\max 20)\end{array}$ & & \\
\hline Pt1 & 4 & 36 & 11 & 10 & 6 & 0 & 0 & 7 \\
\hline $\mathrm{Pt} 2$ & 9 & 13 & 0 & 10 & 8 & 4 & 1 & 2 \\
\hline $\mathrm{Pt} 3$ & 19 & 44 & 11 & 10 & 0 & 0 & 5 & 4 \\
\hline $\mathrm{Pt} 4$ & 10 & 27 & 4 & 7 & 15 & 4 & 3 & 4 \\
\hline $\mathrm{Pt} 5$ & 49 & 41 & 11 & 10 & 3 & 0 & 3 & 4 \\
\hline Pt6 & 47 & 48 & 11 & 10 & 1 & 0 & 6 & 4 \\
\hline
\end{tabular}

** The presence of peripersonal neglect was revealed by means of the Standard Battery for the Evaluation of Hemineglect (Pizzamiglio et al., 1989). Patients who scored below the cut-off on at least two of these four tests were considered to have visuospatial neglect (Pizzamiglio et al., 1989). Letter Cancellation test: cut-off is a difference of 4 (or more) omissions between the right and the left side; Line Cancellation: cut-off is $>2$ omissions on the left side; Wundt-Jastrow Area Illusion Test: cut-off is a difference $>2$ between unexpected answers (i.e., responses in the direction opposite to the illusory effect observed in healthy individuals) given for left-oriented minus right-oriented stimuli; Sentence Reading Test: Hits are the number of correctly read sentences. The cut-off is one or more sentences read incompletely on the left side.

*** The presence of personal neglect was revealed by means of a standardized test (Zoccolotti et al., 1991) that required the use of three objects (eyeglasses, a razor or powder and a comb) in the body space. For each object, the examiner assigned a score based on the asymmetry in performance of the patient in the left and right space $(0=$ no asymmetry, $3=$ maximum asymmetry). 
Table 4 Means and standard deviations of recognition score (R), localization score (L) and leftright inversion error score (LRI-error) in healthy control participants group (C) and braindamaged patients (BDP) group

\begin{tabular}{lr|c|c|c}
\hline & & $\mathbf{R}$ & $\mathbf{L}$ & LRI-error \\
\hline FP's score & $6 / 9$ & $2 / 9$ & $4 / 4$ \\
\hline C & & & \\
\hline mean & 8.53 & 6.23 & 0.7 \\
\hline s.d. & 0.96 & 1.69 & 0.2 \\
\hline & & & \\
\hline
\end{tabular}

